\title{
Dose Adjustment- An Important Issue in Critical Care
}

\author{
Dr. M. C. Joshi MD \\ Pharmacology Department, Indrapratha Apollo Hospital, New Delhi, India
}

(Received 28 October 2005 and accepted 12 December 2005)

\begin{abstract}
There is at times marked variability in drug responsiveness especially in critically ill patients admitted in the Intensive care units. In order to obtain therapeutic effectiveness with in pharmacokinetic parameters related to therapeutic dose, it is always desirable to monitor and to maintain drug dose adjustment in such a way especially in presence of organ failure like renal failure, hepatic failure or any other clinical situation necessitating Therapeutic Drug Monitoring (TDM) so that one can use safe and effective drug therapy with least toxicity due to inaccurate and invalid drug doses.
\end{abstract}

KEY WORDS: Drug, Dose adjustment, Patient's care, Adverse drug reaction.

\section{INTRODUCTION:}

Critically ill adults and children who require comprehensive, specialized care by the members of the ICU Care Team and continuous care including monitoring, assessment, evaluation and treatment of their life-threatening conditions and who are at risk of serious complications should be treated in Intensive Care unit (ICU). Care in the ICU is provided by a multidisciplinary ICU Care Team, which is composed of specially trained physicians, nurses, and other professionals. Each professional brings his or her particular expertise to the team, collaborating on a plan of care and treatment for each patient, based upon his or her individual needs and conditions.

The increasing use of high-tech drugs and sophisticated drug delivery methods would seem to make the ICU a prime area for specialist pharmacy practitioners. Montazeri and Cook showed the benefits of the pharmacist in a multidisciplinary ICU set-up in Canada $^{1}$, and cost savings from having pharmacist input to a medical ICU in the US have also been demonstrated $^{2}$. Leape and colleagues showed a lower rate of adverse drug events associated with pharmacist involvement on prescribing rounds in a medical ICU in the US $^{3}$. The benefits of specialist renal pharmacists have been documented ${ }^{4}$. In a multicentric study in Australia out of 29,269 critically ill patients admitted during the study period, 1738 (5.7\%) had acute renal failure during their ICU stay, including 1260 that were treated with $\mathrm{RRT}^{5}$. Changes in liver perfusion may have a substantial influence on the pharmacokinetics of drugs with flowcontrolled metabolism ${ }^{6}$.

\section{DISCUSSION:}

The kidneys have important physiological functions and play a major role in the excretion of drugs, hormones, and xenobiotics. The liver plays a central role in metabolizing most drugs, which usually require biotransformation for pharmacologic activity or excretion. Drug bioavailability is also controlled by the liver's capacity to clear the drug from the circulation. This depends on both hepatic blood flow and the efficiency of drug removal by hepatocytes (extraction ratio). If the latter is very high, drug clearance primarily depends on hepatic blood flow (e.g. propranolol, lidocaine), whereas flow has relatively little effect on drugs that are slowly cleared by the liver (e.g. theophylline, warfarin, diazepam). Most drugs are cleared at intermediate rates, which are affected by alterations in both hepatic flow and extraction capacity.

Corresponding Author: Dr. M C Joshi, Clinical Pharmacologist, Indrapratha Apollo Hospital, New Delhi110025, India, Email: drjoshimukesh@rediffmail.com 
Both the organs kidney and the liver are vital to maintain the normal drug pharmacokinetics. In ICU patients the physiological functions of kidney and liver is depressed therefore pharmacokinetic alterations occurs in drugs metabolism and excretion and appropriate dose of any drug should be selected on the basis of renal and liver function tests.

\section{Laboratory evaluation of renal function:}

Kidney function is assessed by a variety of tests \& procedures that can be done to evaluate how well kidneys are functioning. These tests are done on urine as well as blood samples. Many conditions can affect the ability of the kidneys to carry out their vital function. The extent of loss of renal function is judged by calculating creatinine clearance which is a useful measure of glomerular filtration rate. According to reduction in creatinine clearance value the patients are categorized into mild, moderate and severe renal impairment.

The standard indicators of renal function are serum levels of urea nitrogen and creatinine; this ratio is normally about $10: 1$. This ratio may increase when renal perfusion or urine flow is decreased, as in urinary tract obstruction or dehydration. Because serum urea nitrogen levels are more affected by these and other factors (e.g. nitrogen intake, catabolism, and use of corticosteroids) than are creatinine levels, the most reliable single indicator of glomerular function is serum levels of creatinine. For example Serum creatinine increasing from 0.5 $\mathrm{mg} / \mathrm{dl}$ to $1 \mathrm{mg} / \mathrm{dl}$ represents a $50 \%$ reduction in GFR.

Serum creatinine is a byproduct of creatinine metabolism in muscle. It is filtered in the glomeruli, but not reabsorbed in the tubules. Therefore, blood values depend closely on GFR. Normal creatinine level is proportional to muscle mass. For example: small woman - $0.5 \mathrm{mg} / \mathrm{dl}$, man - $1.0 \mathrm{mg} / \mathrm{dl}$ and muscular man - $11.4 \mathrm{mg} / \mathrm{dl}$.

- If value doubles, GFR and renal function probably have fallen to half of normal state.

- If value triples, it suggests $75 \%$ loss of renal function.

- Values of $10 \mathrm{mg} / 100 \mathrm{ml}$ suggest $90 \%$ loss of function.

Creatinine Clearance: For 24-hours urine. Normal results: $90-139 \mathrm{ml} / \mathrm{min}$ for Adults and 80-125 $\mathrm{ml} / \mathrm{min}$ for females.

\section{Creatinine Clearance (Cr.CL) Calculation}

The endogenous creatinine clearance (Cr.CL) in milliliter per minute estimates the glomerular filtration rate. A 24 hours urine collection is usually obtained. The procedure for collecting the a timed urine specimen should be explained carefully so that the parent or patient understands fully the rationale of first emptying the bladder (discarding that urine) and noting the time and putting all urine subsequently voided into the collection receptacle, including the last void, 12 or 24 hours later. Reliability of the 24 hours collection can be checked by measuring the total 24 hours creatinine excretion in the specimen. Total daily creatinine excretion (Creatinine Index) should be in the range of $14-20 \mathrm{mg} / \mathrm{kg}$ creatinine indices on either side of this range suggests collections that were either inadequate or excessive. Calculations by the following formula requires measurements of plasma creatinine $\left(\mathrm{P}_{\mathrm{Cr}}\right) \mathrm{mg} / \mathrm{ml}$, urine creatinine $\left(\mathrm{U}_{\mathrm{Cr}}\right)$ in $\mathrm{mg} / \mathrm{ml}$, and urine volume (V) expressed as $\mathrm{ml} / \mathrm{min}$.

$$
\text { Cr.CL }=\mathbf{U}_{\mathrm{Cr}} \times \mathrm{V} / \mathbf{P}_{\mathrm{Cr}}
$$

However, as 24 hour urine based creatinine clearance measurements are time consuming and inaccurate, the parameter most often used to estimate renal function in clinical practice is the serum creatinine concentration. An isolated serum creatinine concentration is unsatisfactory for this purpose as it depends on creatinine production, which is related to muscle mass, as well as on renal elimination of creatinine. A variety of formulae and nomograms have been devised to assist clinicians in predicting the glomerular filtration rate from a stable serum creatinine concentration and the patient's sex, age and weight (or height) ${ }^{7}$. For the purpose of doses adjustment and cautions, the British National Formulary describes renal impairment as "mild", "moderate" or "severe" and the following ranges of GFR have been used to define these terms (Mild: 20-50 ml/min; Moderate: 10-20 ml/min and Severe: $<10 \mathrm{ml} / \mathrm{min}$ ).

\section{Cockcroft \& Gault Equation $^{8}$}

Creatinine clearance $=(140$-age $) \times$ Body weight (Kg) / Serum creatinine (mg/dl) x 72

For female: Multiply above result by 0.85

Note: This formula should be used for steady state creatinine values only. The formula was tested against 24-hour collection and DTPA clearance and had a good correlation of $0.82^{9,10}$. The value of this measure is that it requires only measurement of serum creatinine, and can be calculated by hand. 
$\underline{\text { Jelliffe } R W^{11}}$

Creatinine clearance $=(114-(0.8 \times$ Age $)) /$ S.cr x BSA $/ 1.73 \mathrm{~m}^{2}$

Female: Multiply above result by 0.9 ; S.crserum creatinine in $\mathrm{mg} / \mathrm{dl}$; BSA is body surface area in $\mathrm{m}^{2}$.

\section{Simplified 4-variable MDRD study formula ${ }^{12}$} $\mathrm{GFR}=186.3 \times(\mathrm{S} . \mathrm{Cr})^{-1.154} \times$ (age in years) $^{-}$ ${ }^{0.203} \times 1.212$ (if patient is black) $\mathrm{x} 0.742$ (if female).

It may represent the most accurate choice for patients with chronic kidney disease. Note that the units used in the United States are milligrams per deciliter (mg/dl). To convert to international units (micromoles per liter) multiply the creatinine (in $\mathrm{mg} / \mathrm{dl}$ ) by 88 . Thus a serum creatinine of $2 \mathrm{mg} / \mathrm{dl}$ is the same as 176 micromoles per liter.

\section{Assessment of Liver Function:}

Unlike in renal disease, where creatinine clearance estimates provide a reasonable guide to alterations in drug dosage requirements, indicators of hepatic disease, such as elevated liver enzymes, low serum albumin concentrations and clotting abnormalities, cannot be directly related to drug clearance.
Nevertheless, patients with severe cirrhosis will often need reduced doses of hepatically cleared drugs to avoid toxicity. The altered response to drugs in liver disease could be due decreased metabolizing capacity of the hepatocytes, impaired biliary elimination due to biliary obstruction (e.g rifampicin accumulates in obstruction jaundice), impaired hepatic blood flow leading to an increase in bioavailability caused by a reduction in first pass metabolism (e.g bioavailabilities of morphine and labetalol have been reported to double in patients with cirrhosis), decreased protein binding and increased toxicity of drugs highly bound to plasma protein (e.g. phenytoin, warfarin) due to impaired albumin production, altered volume of distribution of drugs due to increased extracellular fluid, oedema and ascitis in liver disease may be exacerbated by drugs that cause fluid retention (e.g. acetylsalicylic acid, ibuprofen, prednisolone, dexamethasone).

C.G. Child and J.G. Turcotte first proposed the scoring system for severity of liver diseases in 1964, which was modified by Pugh in 1972 . He replaced Child's criterion of nutritional status with the prothrombin time or INR, and thus eliminated the most subjective part of the score.

\section{A. Child-Pugh system ${ }^{13}$}

\begin{tabular}{lccc}
\hline Hepatic Parameters & Points Scored for Observed Findings & \\
& $\mathbf{1}$ & $\mathbf{2}$ & $\mathbf{3}$ \\
Encephalopathy grade* & none & 1 or 2 & 3 or 4 \\
Ascites & absent & slight & moderate \\
Serum bilirubin, mg/dL & $<2$ & 2 to 3 & $>3$ \\
Serum albumin, g/dL & $>3.5$ & 2.8 to 3.5 & $<2.8$ \\
Prothrombin time, sec prolonged & $<4$ & 4 to 6 & $>6$ \\
\hline
\end{tabular}

Note: Encephalopathy grade*_

- Grade 0: normal consciousness, personality, neurological examination, EEG

- $\quad$ Grade 1: restless, irritable/agitated, tremor, impaired handwriting, 5 cps waves

- $\quad$ Grade 2: lethargic, time-disoriented, inappropriate, asterixis, ataxia, slow triphasic waves

- Grade 3: somnolent, stuporous, disoriented, hyperactive reflexes, rigidity, slower waves

- $\quad$ Grade 4: unarousable coma, no personality/behavior, decerebrate, slow 2-3 cps delta activity Assessment:

1. Class A or mild: If 5 or 6 points; Good operative risk

2. Class B or moderate: If 7 to 9 points; Moderate risk

3. Class $C$ or severe: If 10 to 15 points; Poor operative risk

(Developed for surgical evaluation of alcoholic cirrhotic) 
Patients with a score of 10 or more (in the Class C category) have a prognosis with 1-year survival being about 50\%. Patients with Class A or $\mathrm{B}$ have a better prognosis of 5-years, with a survival rate of $70 \%-80 \%$. Additional poor prognostic indices include refractory ascites, albumin $<3.2 \mathrm{gm} / \mathrm{l}$, and a recent episode of SBP (spontaneous bacterial peritonitis). All individually are associated with a one-year survival of $50 \%$ or less.

\section{B. Maddrey discriminant function $(d f)^{14}$}

Discriminant Function_ $=4.6 \times$ [patient's PT control PT] + total bilirubin (mg/dL).

Interpretation of the discriminant Function (df) values in patients with acute alcoholic hepatitis was that the disease was not severe if $\mathrm{df}<54$, was severe if 55 to 92, and probably lethal if 93 or more and untreated.

The df was modified in a later study by Carithers et al (1989) to use the prolongation of prothrombin time above normal control values and to divide the serum bilirubin by 17.1 to give $\mathrm{mmol} / \mathrm{L}$. Patients with modified df values of 32 or more were entered into a study of methyl prednisolone treatment, corresponding to Maddrey $d f$ values of approximately 106 .

\section{The Mayo End-Stage Liver Disease (MELD) score $e^{15}$}

It is a marker of disease severity and mortality in persons with chronic alcoholic liver disease. It has also been assessed as a good predictor of short-term mortality in persons with alcoholic hepatitis $^{\mathbf{1 3}}$.

$M E L D$ score $=3.8 \times \log _{\mathrm{e}}(\mathrm{TBR})+11.2 \mathrm{x}$ $\log _{\mathrm{e}}(\mathrm{INR})+9.6 \mathrm{x} \log _{\mathrm{e}}($ creatinine $)$

(TBR - total bilirubin in mg/dl and creatinine in $\mathrm{mg} / \mathrm{dl})$

\section{Hepatic contribution to the elimination of the compound}

- For no hepatic contribution to the elimination of the compound: If greater than $90 \%$ of the dose is excreted in the urine as unchanged drug, hepatic impairment would not be expected to have a significant effect on elimination.

- For limited $(<20$ percent $)$ hepatic elimination: Wide Therapeutic RangeBecause greater than $80 \%$ of the dose is excreted in the urine as unchanged drug, hepatic impairment would not be expected to lead to unsafe systemic exposure. Narrow Therapeutic Range- Because the usual doses of the drug are close to doses that can cause adverse effects, and there is in-vitro or invivo evidence of hepatic contribution to the elimination, hepatic impairment could lead to an increased exposure and possibly an increase in adverse effects. Patients with impaired liver function may require reduced doses of or longer dosing intervals. If drug is used, close monitoring of patients with impaired liver function is important.

- For extensive (> 20 Percent) hepatic elimination: Wide Therapeutic RangeBecause there is in-vitro or in-vivo evidence of extensive hepatic contribution to the elimination, hepatic impairment would be expected to have significant effects on the pharmacokinetics. Caution should be exercised during the use. Patients with impaired liver function may require reduced doses or longer dosing intervals. Narrow Therapeutic Range- Because there is in-vitro or in-vivo evidence of extensive hepatic contribution to the elimination, hepatic impairment would be expected to have significant effects on pharmacokinetics. Drug should be avoided or used with great caution in this patient population.

- For unknown hepatic elimination: Consider the compound as extensively metabolized and use the above format.

\section{CONCLUSION:}

Ideally in planning 'dose regimen' drug dosage adjustment is of paramount importance in all critically ill patients with organ failures. In treating such critically ill patients in 'Intensive care units', drug treatment should be so instituted looking forward to their organ display using a battery of selected tests as indicated in order to achieve steady state plasma concentration required within a known and safe effective therapeutic range.

\section{REFERENCES:}

1. Montazeri M, Cook DJ. Impact of a clinical pharmacist in a multidisciplinary intensive care unit. Crit Care Med 1994;22:1044-8.

2. Baldinger SL, Chow MSS, Gannon RH, Kelly ET. Cost savings from having a clinical pharmacist work part-time in a medical intensive care unit. Am J Health Syst Pharm 1997;54:2811-4. 
3. Leape LL. Pharmacist participation on physician rounds and adverse drug events in the intensive care unit. JAMA 1999;282:26770.

4. MacLean D. Defining a niche for the renal pharmacist. Hosp Pharm 1994;1:20-2.

5. Uchino $\mathrm{S}$ et al. Acute renal failure in critically ill patients: a multinational, multicenter study. JAMA 2005 Aug 17;294(7):813-8.

6. Koppel C, Pohle S et al. Rapid determination of functional liver plasma flow in ICU patients by a modified hepatic D-sorbitol plasma clearance method.

7. SA Smith. Estimation of glomerular filtration rate from the serum creatinine concentration. Postgraduate Medical Journal 1988;64:204-208

8. Cockcroft DW, Gault MH. Prediction of creatinine clearance from serum creatinine. Nephron 1976;16(1):31-41.

9. Gault $\mathrm{MH}$, Longerich LL, Harnett JD, Wesolowski C. Predicting glomerular function from adjusted serum creatinine. Nephron 1992;62:249-256.
10. Rose BD. Clinical Physiology of Acid-Base and Electrolyte Disorders Fourth Edition 1994. McGraw-Hill, Inc., New York, USA.

11. Jelliffe RW. Estimation of creatinine clearance when urine cannot be collected. Lancet 1971;1:975-6.

12. Levey AS, Bosch JP, Lewis JB, et al. A more accurate method to estimate glomerular filtration rate from serum creatinine: a new prediction equation. Modification of Diet in Renal Disease Study Group. Ann Intern Med 1999;130(6):461-70.

13. Pugh RNH, Murray-Lyon IM, Dawson JL, Pietroni MC, Williams R, Transection of the oesophagus for bleeding oesophageal varices. Brit J Surg 1973;60:646-9.

14. Maddrey WC, Boitnott JK, Bedine MS et al. Corticosteroid therapy of alcoholic hepatitis. Gastroenterology 1978;75:193-9.

15. Srikureja W, Kyulo NL, Runyon BA et al. MELD (Mayo end-stage liver disease) score is a better prognostic model than ChildTurcotte-Pugh score or Discriminant Function score in patients with alcoholic hepatitis. J Hepatol 2005 May;42(5):700-6. 